\title{
Colorimetric Research of the Self-Luminous Objects in Software and Hardware Environments by the Method of Multidimensional Scales Implementation
}

\author{
SaukovaYauheniya* \\ Belarusian National Technical University, Minsk, Republic of Belarus
}

*Corresponding Author: SaukovaYauheniya, PhD in Engineering sciences, Belarusian National Technical University, Minsk, Republic of Belarus

\begin{abstract}
The experiment on the accuracy evaluation of colorimetric measurements in software and hardware environments carried out by the Research Laboratory of Optical Electronic Instrument Engineering within the framework of the State Program of scientific research "Electronics and Photonics" is described. The experiment was conducted in the accredited testing laboratory of the Public Corporation "Rudensk" (Rudensk, Republic of Belarus) which uses the reference measurement procedure. Under the conditions of repeatability and intermediate precision the color and chromaticity coordinates of the created samples were determined by their multiple digital registration using vision system (digital camera) to determine the capabilities of the methodology from the standpoint of building a multidimensional conditional scale.
\end{abstract}

Keywords: colorimetry, digital image, chromaticity, color scale, accuracy

\section{INTRODUCTION}

Colorimetry is a color measurement performed in accordance with the adopted system of conventions (international agreements) [1]. Basic colorimetry [2] includes standard models of observation conditions and is used in the control of products in medicine, chemistry, printing, painting, and automotive industry [3-5]. High colorimetry "includes the methods for assessing the perception of color stimulus presented to the observer in a complex environment that we observe in everyday life» $[2,6]$, i.e. in real conditions of observation and is used for example in the development of lighting scenarios. High resolution colorimetry is a methodology of qualitative and quantitative methods for studying the color characteristics of self-luminous and non-self-luminous objects based on digital registration technologies and digital images processing which allows to provide reliable results with a given level of reliability. Sometimes we can have situations when any of the color channels in the digital image are in saturation or at noise level and the color range of the recording device is sharply narrowed, causing distortion of the measurement information. The developed method provides new measurement capabilities as it allows by repeatedly registering the surface of the object to calculate the brightness values in three color channels differing by several orders of magnitude by comparing the brightness of each point of the object with the reference brightness of reference samples and performing mathematical transformations using conventional scales which will calculate the color coordinates in the standard color space for each control point of the object. Research Laboratory of Optoelectronic Instrumentation (Belarusian National Technical University, Minsk, Republic of Belarus) develops the methodology as a part of the tasks of the State research Program "Electronics and Photonics».

The main provisions of colorimetry in hardware and software environments are formulated as follows:

- Digital image is the result of elements color gamut convolution of the information and measurement channel including a light source, illuminated object, matrix photo detector, software, display device. Each element of the channel is a potential object of research depending on the task for example evaluation of color rendering of the light source, a control of illuminated object color and brightness properties, evaluation of system software capabilities, digital camera adjustment, 
Colorimetric research of the self-luminous objects in Software and Hardware environments by the Method of multidimensional scales implementation

information display device setting, etc. Thus a digital image is an information model of one of the elements provided that all other elements are validated [7].

- Determination of color characteristics in discrete vision systems with an arbitrary number of input and output values is based on the metamerism phenomena, statistical redundancy and quantization effects and consists in averaging the digital image signals over a certain area in the context of the "space-time" factor. Therefore, the methodology is applicable to non-point (extended) objects.

- Principles and methods of objects photometric and colorimetric characteristics measurements in software and hardware environments are based on digital registration of the object and certified reference samples by a matrix photo detector. Optoelectronic transformations in the information measuring channel and processing of the received digital images allow to build intensity calibration dependences. These are the dependences of the digital image intensity on the brightness of reference samples and exposure time for three color channels which make it possible to determine the brightness and color at the object control point from it digital image. Certified (reference) samples provide metrological traceability by constructing a multidimensional conventional scale and are non-point primary or secondary emitters with uniformly distributed brightness (reflection coefficient) [8].

- The definitional uncertainty characterizes the smallest value scattering region in the multidimensional functional space due to limited detail in the definition of color as a subjective sensation and an Athenian vector quantity. The definitional uncertainty depends on the nominal quantization steps reproduced by conventional digital image scales which in three-dimensional color spaces in the limiting case are the McAdam ellipses [9].

- The result of measuring chromaticity coordinates as a vector quantity is described by a vector column (expectation) and a set of covariance matrices (uncertainty). So we carry out metrological modeling of the colorimetric measurements results in software and hardware environments in discrete systems with an arbitrary number of input and output values on the basis of modular ascending and descending approaches with building of mathematical expectation models of measurement results and models for dispersion (uncertainty and covariance), integration of uncertainty components taking into account the entropy of measurement information.

The method of numerical reference multidimensional colorimetric scale modeling is considered in detail in [10]. It consists in creating a set of certified reference samples (non-point emitters) related to a particular sector of the color palette and forming at digital registration with incrementally increasing exposure time vectors in the color space XYZ. These vectors originate from the zero point of space and intersect the plane of the color locus of the chromaticity graph forming a geometric place of its chromaticity points. This procedure allows providing the condition of uniformity of measurements. In [10], the authors establish regularities of mapping calibration dependencies in the XYZ space for its division (zoning) into sectors by numerical simulation. Thus, we are able to determine the desired sector of the color space in the measurement process and reduce the methodological component of uncertainty. The authors carried out the division of the palette into sectors on the principle of predominance of $\mathrm{R}$ (red), $\mathrm{G}$ (green) and $\mathrm{B}$ (blue) components available in descending order. Sector I RGB; Sector II - RBG; Sector III - GBR; Sector IV - GRB; Sector V - BRG; Sector VI - BGR. We determined the intensities R, G, B (color coordinates) of each element within the sectors based on averaging the intensities over an area of approximately $60 * 60$ to $128 * 128$ pixels. Then we transformed the $\mathrm{RGB} \rightarrow \mathrm{XYZ}$ color coordinates and calculated the chromaticity coordinates. We took three cells of the same color from each sector from light to darker the boundaries between sectors in space were determined XYZ by the calculations.

This division allows you to determine the chromaticity zone in the visible range of reference samples (primary emitters) that are used to build calibration dependencies. Further, we solved the problem of optimization of quantitative and qualitative composition of reference samples depending on the required photometric and colorimetric characteristics. We used the developed computer program "Photon" to zone the XYZ color space in an automated mode [11].

\section{HYPOTHESIS}

The modular principle of step-by-step graphic data processing is the basis of the methodology for the transition from photometric measurement of scalar quantities to colorimetric measurement of vector 
Colorimetric research of the self-luminous objects in Software and Hardware environments by the Method of multidimensional scales implementation

quantities. They highlight control points or areas in the method of measuring the brightness of a selfluminous object on the surface of the object under study. Then it is necessary to place the reference light sources with a known brightness and with the help of a matrix photo detector to carry out multiple registrations of self-luminous object and reference light sources with incrementally increasing exposure time. Then you need to perform computer processing of the digital images to determine the intensity of the digital image areas and brightness in the control area of the selfluminous object by the formula [12]:

$L=\left(N-N_{01}\right) \frac{\left(L_{02}-L_{01}\right)}{N_{02}-N_{01}}+L_{01}$,

Where $N$-averaged intensity of the corresponding area of the digital image, relative units:

$N=\frac{1}{n} \sum_{i=1}^{n} N_{i}$

Where $N_{i^{-}}$intensity of $i$-thdigital image element, relative units;

$L_{01}, L_{02}$-brightness values of reference light sources, $\mathrm{cd} / \mathrm{m}^{2}$;

$N_{01}, N_{02}$-averaged intensity of digital image areas of reference light sources, relative units.

The obtained calibration dependences are shown in figures 1 and 2 .

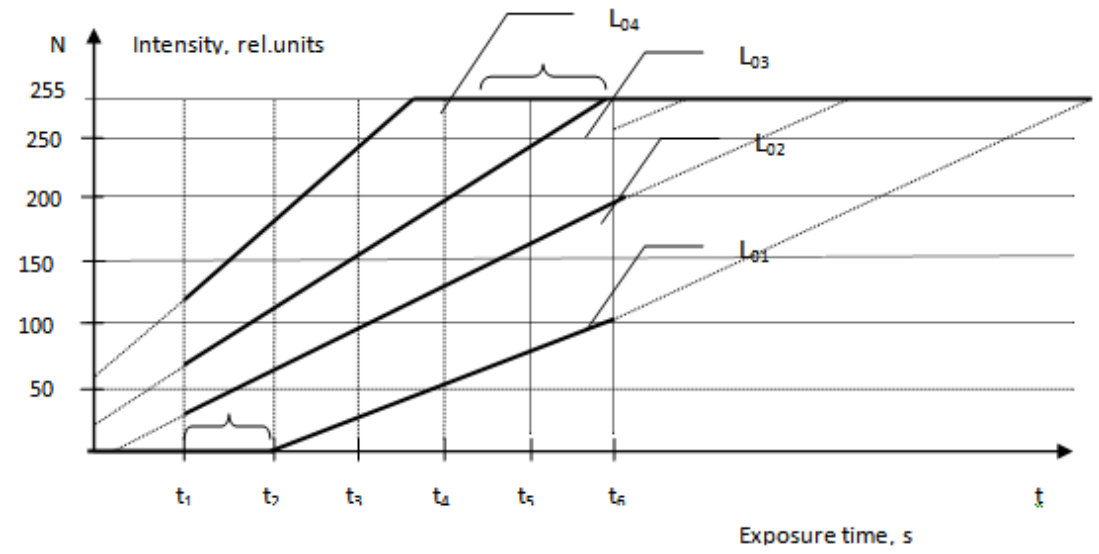

Figure1. Calibration dependences of digital image area intensity on exposure time

In figure 1 , the exposure time is plotted along the horizontal axis, the intensity of the digital image areas corresponding to the reference light sources is shown on the vertical axis. Figure 3 shows a dependency coupling where the brightness of the reference light sources, $\mathrm{cd} / \mathrm{m} 2$, is plotted along the horizontal axis. The intensity of the digital images areas of corresponding to the reference light sources for different exposure times is shown on the vertical axis. The use of reference light sources allows to determine the brightness of the self-luminous object in the controlled areas by the characteristic dependences. Thus we are able to expand the dynamic range of measurements [13].

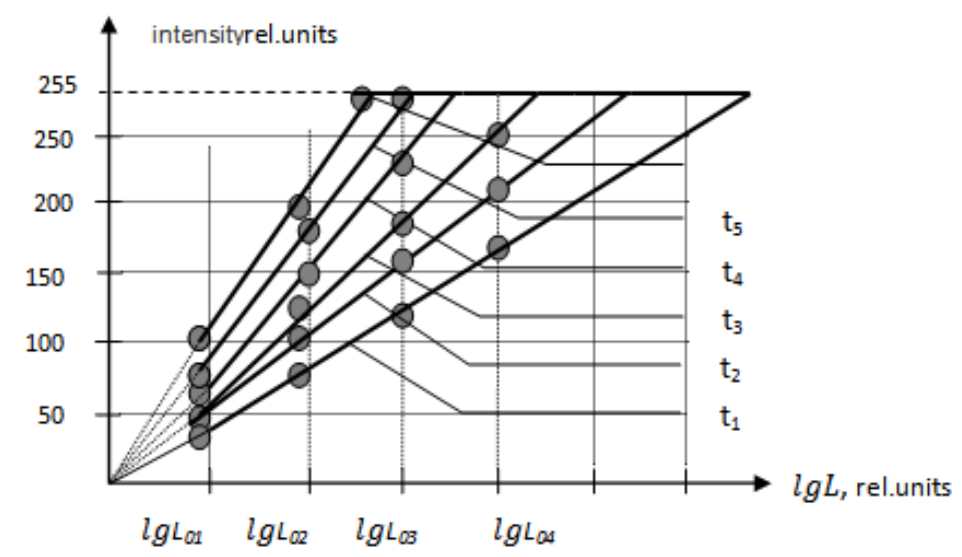

Figure2. Calibration dependences of digital image intensity on the brightness of reference light sources for different exposure time 
Colorimetric research of the self-luminous objects in Software and Hardware environments by the Method of multidimensional scales implementation

So the intensity of the digital image within the color gamut also increases with increasing the exposure time. This active range is typically about $60 \mathrm{~dB}$.

Figure 3 shows the characteristic dependences of the output signals of the CCD array of the photo detector corresponding to images made at shutter speeds $\mathrm{t} 1<\mathrm{t} 2<\mathrm{t} 3$ for one of the color channels. The points L01 and L01 correspond to the brightness values of the color targets in this color channel. The dynamic range of the matrix photo detector is limited by the region of noise (point $\mathrm{N} 1$, dotted line) and saturation (pointsLsat1, Lsat2, Lsat3), the areas II and II illustrate the expansion of the dynamic range by mathematical modeling.

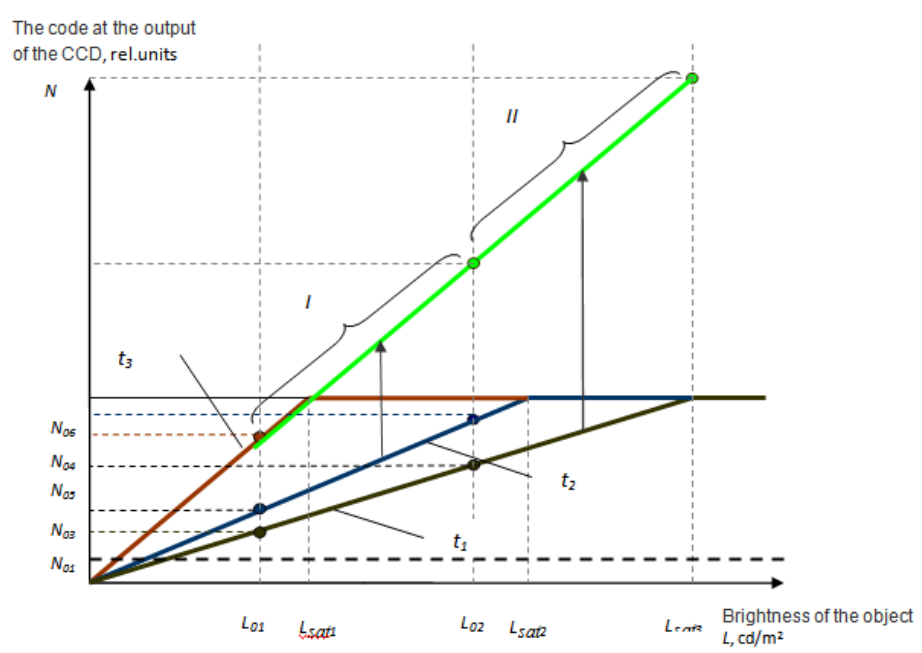

Figure4. The calibration dependence of the intensity of the color channels of brightness

During the planning phase of the experiment, the camera must be tested in the laboratory to determine the number of defects in the photosensitive field of the matrix, the uneven sensitivity of the image field, the saturation signal, the dark signal level and the dynamic range width by performing a series of calibration frames if the manufacturer has not specified this information).

For each curve in the three color channels, we expected large-scale conversion factorsK12, K23:

$\mathrm{K} 12=\frac{N_{2}}{N_{1}} ; \quad \mathrm{K} 23=\frac{N_{3}}{N_{2}}$,

whereN1 $=\mathrm{N} 1(\mathrm{~L})=\mathrm{K} 12 \mathrm{~L} ; \quad \mathrm{N} 2=\mathrm{N} 2(\mathrm{~L})=\mathrm{K} 23 \mathrm{~L}$

Then We paired the dependencies to calculate the brightness of the control point of the object withinLsat $2 \leq \mathrm{L} \leq \mathrm{Lsat} 3$,

$$
\mathrm{L}= \begin{cases}v_{1}, & \text { by } L<L_{\text {sat1 } 1} \\ V_{2} \cdot K_{12}, & \\ V_{3} \cdot K_{12} \cdot K_{23}, & \text { by } L_{\text {sat } 1}<L<L_{\text {sat } 2}\end{cases}
$$

Relative errors were determined by the formulas:

$$
\frac{\Delta L_{1}}{L_{1}}=\frac{\Delta N_{1}}{N_{1}} ; \frac{\Delta L_{2}}{L_{2}}=\frac{\Delta N_{2} \cdot K_{12}}{N_{2} \cdot K_{12}} ; \frac{\Delta L_{3}}{L_{3}}=\frac{\Delta N_{3} \cdot K_{12} \cdot K_{23}}{N_{3} \cdot K_{12} \cdot K_{23}} \ldots
$$

Since the value $\frac{\Delta N_{i}}{N_{i}}$ is the same in all ranges the relative error $\frac{\Delta L_{i}}{L_{i}}$ also did not change.

We calculated the exposure from the expression:

$\mathrm{H}=\mathrm{E} \Delta \mathrm{t}$, 
Colorimetric research of the self-luminous objects in Software and Hardware environments by the Method of multidimensional scales implementation

WhereE-illumination at the point of the object,

$\Delta \mathrm{t}$-exposure time.

For the same point on the object:

$\mathrm{H} 1=\mathrm{E} \Delta \mathrm{t} 1 ; \mathrm{H} 2=\mathrm{E} \Delta \mathrm{t} 2$.

Then

$\mathrm{E}=\frac{H_{1}}{\Delta t_{1}}=\frac{H_{2}}{\Delta t_{2}}$

Therefore,

$\mathrm{H} 1=\mathrm{kH} 2, \mathrm{nk}=\frac{\frac{H_{1}}{H_{2}}}{\Delta t_{2}}$

When converted to light units, the scatter of points relative to the calibration curve was not more than $10 \%$, which shows the possibility of using this method. Thus, the expansion of the range of the measurement method can be carried out by computer processing of digital images.

The experiment on the scale implementation was conducted on the basis of the accredited testing laboratory of public corporation "Rudensk" (Rudensk, Republic of Belarus).We used monitors as reference samples (self-luminous objects).We created uniform color fields on the monitors so that they represent consistent color pairs i.e. they are in the same sector of the XYZ color space. They allowed constructing vectors in the aggregate representing a multidimensional reference scale. Examples of experimental (reference) samples pairs are shown in figure 4.
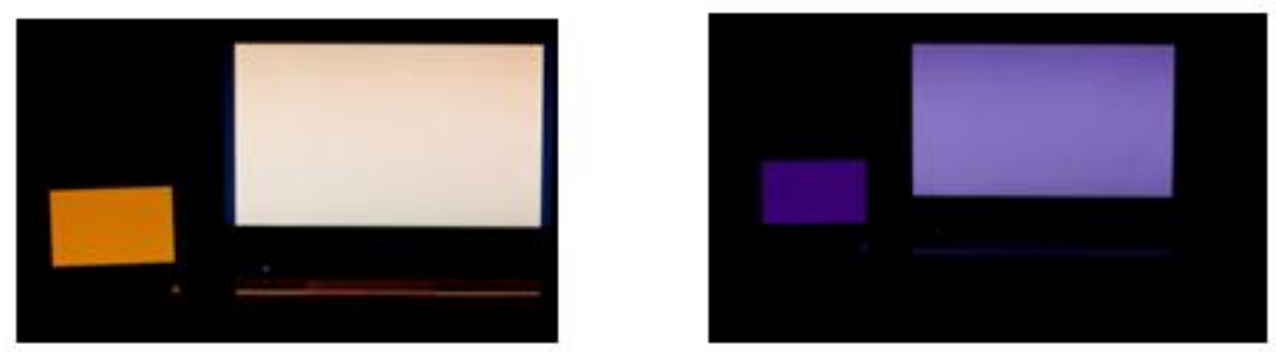

Figure4. Reference samples for the experiment

The lighting characteristics of the samples were determined using a measuring system including a colorimeter C1210 with a colorimetric head "Lichtmesstechnik GMBH Berlin" (Germany), a source of the type A with a control filters set. Measurement range of chromaticity coordinates is for $\mathrm{x}$ : from 0,0039 to 0 , and 7347; for $\mathrm{y}$ : from 0,0048 to 0,8338 . Limits of permissible absolute error of chromaticity coordinates measurement are $\Delta \mathrm{x}=\Delta \mathrm{y}=0,007$. Diameter of the photosensitive surface is $60 \mathrm{~mm}$. Minimum display value is $0,01 \mathrm{~lx}$; maximum display value is $600000 \mathrm{~lx}$.

We were shooting reference samples from the same point in space using a semi-professional digital camera Nikon 5100 with optics Nikon $35 \mathrm{~mm}$ f/1.8G AF-S DX Nikon and exposure time $0,1 \mathrm{~s} ; 0,2 \mathrm{~s} ; 0,3$ s. Images were saved in the format RAW. Next, we processed digital images and determined the intensities R, G, B and calculated the color coordinates by formulas [14]:

$\mathrm{X}=2.7689 \cdot \mathrm{R}+1.7517 \cdot \mathrm{G}+1.1302 \cdot \mathrm{B}$

$\mathrm{Y}=1.0000 \cdot \mathrm{R}+4.5907 \cdot \mathrm{G}+0.0601 \cdot \mathrm{B}$

$\mathrm{Z}=0.0565 \cdot \mathrm{G}+5.5943 \cdot \mathrm{B}$

Transformations of color coordinates into chromaticity coordinates were carried out by formulas [15]:

$\mathrm{x}=\frac{X}{X+Y+Z}$;

International Journal of Innovative Research in Electronics and Communications (IJIREC) 
Colorimetric research of the self-luminous objects in Software and Hardware environments by the Method of multidimensional scales implementation

$$
\begin{aligned}
& \mathrm{y}=\frac{Y}{X+Y+Z} \\
& \mathrm{z}=\frac{Y}{X+Y+Z}
\end{aligned}
$$

Mathematical expectations (arithmetic mean values) of chromaticity coordinates and their mean square deviations were found sx, sy, sz [16]:

$s_{x}=\sqrt{\frac{1}{t-1}\left(x_{j}-\bar{x}\right)^{2}}$

$\mathrm{s}_{\mathrm{y}}=\sqrt{\frac{1}{\mathrm{t}-1}\left(\mathrm{y}_{\mathrm{j}}-\overline{\mathrm{y}}\right)^{2}}$

$\mathrm{S}_{\mathrm{z}}=\sqrt{\frac{1}{\mathrm{t}-1}\left(\mathrm{z}_{\mathrm{j}}-\overline{\mathrm{z}}\right)^{2}}$

Where $\mathrm{t}-$ number of values in the aggregate (for this experiment $\mathrm{t}=3$;

$\mathrm{x}_{\mathrm{j}}, \mathrm{y}_{\mathrm{j}}, \mathrm{z}_{\mathrm{j}}-\mathrm{j}$-th chromaticity coordinates;

$\overline{\mathrm{x}}, \overline{\mathrm{y}}, \overline{\mathrm{z}}$ - mathematical expectations of chromaticity coordinates.

The total standard deviation $\mathrm{s} \Sigma$ is [17]:

$\mathrm{s}_{\Sigma}=\sqrt{\mathrm{s}_{\mathrm{x}}^{2}+\mathrm{s}_{\mathrm{y}}^{2}+\mathrm{s}_{\mathrm{z}}^{2}}$

The displacements were calculated according to [18] by the formula:

$\delta_{\mathrm{x}}=\overline{\mathrm{x}}-\mu_{\mathrm{x}}$

$\delta_{\mathrm{y}}=\overline{\mathrm{y}}-\mu_{\mathrm{y}}$

$\delta_{\mathrm{z}}=\overline{\mathrm{z}}-\mu_{\mathrm{z}}$

\begin{tabular}{|c|c|c|c|c|c|c|c|c|c|}
\hline \multirow[t]{2}{*}{ Exposuretime, } & \multicolumn{3}{|c|}{$\begin{array}{l}\text { Color coordinates in the } \\
\text { space RGB }\end{array}$} & \multicolumn{3}{|c|}{$\begin{array}{l}\text { Color coordinates in the space } \\
\mathrm{XYZ}\end{array}$} & \multicolumn{3}{|c|}{$\begin{array}{l}\text { chromaticity } \\
\text { coordinatesBin the space } \\
\text { XYZ }\end{array}$} \\
\hline & $\mathrm{R}$ & G & $\mathrm{B}$ & $\mathrm{X}$ & $\mathrm{Y}$ & $\mathrm{Z}$ & $\mathrm{x}$ & $\mathrm{y}$ & $\mathrm{z}$ \\
\hline \multicolumn{10}{|c|}{ «yellowdark» $\mathrm{x}=0,3920 \mathrm{y}=0,4445 \mathrm{z}=0,1635$} \\
\hline 0,1 & 161 & 150 & 0 & 708,5479 & 423,755 & 262,755 & 0,4012 & 0,4475 & 0,1523 \\
\hline 0,2 & 201 & 210 & 3 & 927,7965 & 1165,2273 & 28,6479 & 0,3803 & 0,4314 & 0,1883 \\
\hline 0,3 & 233 & 229 & 11 & 1058,7252 & 1284,9314 & 74,47 & 0,3981 & 0,4459 & 0,1560 \\
\hline \multicolumn{7}{|l|}{ Meanvalue } & 0,3840 & 0,4355 & 0,1655 \\
\hline \multicolumn{10}{|c|}{ the standard deviations $\mathrm{s}_{\mathrm{x}}=0,026 ; \mathrm{s}_{\mathrm{y}}=0,025 ; \mathrm{s}_{\mathrm{z}}=0,027 ; \mathrm{s}_{\Sigma}=0,045$} \\
\hline \multicolumn{10}{|c|}{ bias $\delta_{x}=0,008 ; \delta_{y}=-0,009 ; \delta_{z}=0,020$} \\
\hline \multicolumn{10}{|c|}{ «yellow light» $\mathrm{x}=0,4229, \mathrm{y}=0,4670, \mathrm{z}=0,1101$} \\
\hline 0,1 & 178 & 172 & 2 & 796,417 & 481,5528 & 303,5528 & 0,4296 & 0,4632 & 0,1072 \\
\hline 0,2 & 215 & 222 & 19 & 1005,6647 & 1235,2773 & 118,8347 & 0,4313 & 0,4681 & 0,1006 \\
\hline 0,3 & 252 & 249 & 39 & 1178,0139 & 1397,4282 & 232,2462 & 0,4126 & 0,4642 & 0,1232 \\
\hline \multicolumn{7}{|c|}{ Meanvalue } & 0,4245 & 0,4651 & 0,1113 \\
\hline \multicolumn{10}{|c|}{ the standard deviations $\mathrm{s}_{\mathrm{x}}=0,027 ; \mathrm{s}_{\mathrm{y}}=0,027 ; \mathrm{s}_{\mathrm{z}}=0,028 ; \mathrm{s}_{\Sigma}=0,046$} \\
\hline \multicolumn{10}{|c|}{ bias e $\delta_{x}=0,016 ; \delta_{y}=-0,019 ; \delta_{z}=0,012$} \\
\hline \multicolumn{10}{|c|}{ «bluedark» $\mathrm{x}=0,1250 \mathrm{y}=0,1030 \mathrm{z}=0,7720$} \\
\hline 0,1 & 0 & 8 & 135 & 166,5906 & 166,5906 & 166,5906 & 0,1241 & 0,1021 & 0,7738 \\
\hline 0,2 & 0 & 21 & 167 & 225,5291 & 106,4414 & 935,4346 & 0,1259 & 0,1041 & 0,77 \\
\hline 0,3 & 0 & 45 & 214 & 320,6893 & 219,4429 & 1199,723 & 0,1264 & 0,1038 & 0,7708 \\
\hline
\end{tabular}

A fragment of intermediate calculations for three pairs of samples is presented in table 1 .

Table1. Experimental data of colorimetric measurements

International Journal of Innovative Research in Electronics and Communications (IJIREC)

Page | 30 
Colorimetric research of the self-luminous objects in Software and Hardware environments by the Method of multidimensional scales implementation

\begin{tabular}{|c|c|c|c|c|c|c|c|c|c|}
\hline \multicolumn{7}{|l|}{ Meanvalue } & 0,1261 & 0,1033 & 0,7715 \\
\hline \multicolumn{10}{|c|}{$s_{x}=0,027 ; s_{y}=0,027 ; s_{z}=0,028 ; s_{\Sigma}=0,047$} \\
\hline \multicolumn{10}{|c|}{$\operatorname{bias} \delta_{\mathrm{x}}=0,011 ; \delta_{\mathrm{y}}=-0,03 ; \delta_{\mathrm{z}}=0,05$} \\
\hline \multicolumn{10}{|c|}{ «bluelight» $\mathrm{x}=0,1326 \mathrm{y}=0,1173 \mathrm{z}=0,7501$} \\
\hline 0,1 & 3 & 16 & 144 & 199,0827 & 199,0827 & 190,776 & 0,1393 & 0,1162 & 0,7445 \\
\hline 0,2 & 6 & 30 & 177 & 269,2098 & 154,3587 & 991,8861 & 0,1310 & 0,1179 & 0,7511 \\
\hline 0,3 & 10 & 56 & 225 & 355,5554 & 216,3319 & 1261,09 & 0,1416 & 0,1169 & 0,7415 \\
\hline \multicolumn{7}{|c|}{ Meanvalue } & 0,1373 & 0,1170 & 0,7457 \\
\hline \multicolumn{10}{|c|}{$\mathrm{s}_{\mathrm{x}}=0,0027 ; \mathrm{s}_{\mathrm{y}}=0,0028 ; \mathrm{s}_{\mathrm{z}}=0,0028 ; \mathrm{s}_{\Sigma}=0,0047$} \\
\hline \multicolumn{10}{|c|}{ bias $\delta_{\mathrm{x}}=0,0047 ; \delta_{\mathrm{y}}=-0,0003 ; \delta_{\mathrm{z}}=-0,0044$} \\
\hline \multicolumn{10}{|c|}{$\langle$ reddark» $\mathrm{x}=0,5104 \mathrm{y}=0,2826 \mathrm{z}=0,2070$} \\
\hline 0,1 & 129 & 25 & 1 & 402,1108 & 334,7335 & 44,9227 & 0,5115 & 0,2811 & 0,2074 \\
\hline 0,2 & 183 & 33 & 4 & 569,0356 & 402,1108 & 24,2417 & 0,5114 & 0,2829 & 0,2057 \\
\hline 0,3 & 244 & 47 & 6 & 764,7227 & 460,1235 & 36,2213 & 0,5153 & 0,2894 & 0,1953 \\
\hline \multicolumn{7}{|c|}{ Meanvalue } & 0,5127 & 0,2845 & 0,2028 \\
\hline \multicolumn{10}{|c|}{$s_{x}=0,024 ; s_{y}=0,024 ; s_{z}=0,025 ; s_{\Sigma}=0,042$} \\
\hline \multicolumn{10}{|c|}{$\operatorname{bias} \delta_{x}=0,066 ; \delta_{y}=0,019 ; \delta_{z}=-0,042$} \\
\hline \multicolumn{10}{|c|}{ «redlight $» \mathrm{x}=0,5506 \mathrm{y}=0,3219 \mathrm{z}=0,1276$} \\
\hline 0,1 & 140 & 34 & 4 & 451,7246 & 451,7246 & 64,0786 & 0,5564 & 0,3220 & 0,1216 \\
\hline 0,2 & 194 & 44 & 6 & 621,0226 & 396,3514 & 36,0518 & 0,5489 & 0,3212 & 0,1299 \\
\hline 0,3 & 253 & 55 & 8 & 805,9168 & 505,9693 & 47,8619 & 0,5515 & 0,3222 & 0,1263 \\
\hline \multicolumn{7}{|l|}{ Meanvalue } & 0,5523 & 0,3218 & 0,1259 \\
\hline \multicolumn{10}{|c|}{$\mathrm{s}_{\mathrm{x}}=0,025 ; \mathrm{s}_{\mathrm{y}}=0,025 ; \mathrm{s}_{\mathrm{z}}=0,026 ; \mathrm{s}_{\Sigma}=0,044$} \\
\hline \multicolumn{10}{|c|}{$\operatorname{bias} \delta_{\mathrm{x}}=0,017 ; \delta_{\mathrm{y}}=-0,001 ; \delta_{\mathrm{z}}=-0,017$} \\
\hline
\end{tabular}

The intensity dependences in the color channels for these samples are shown in figure 6 .

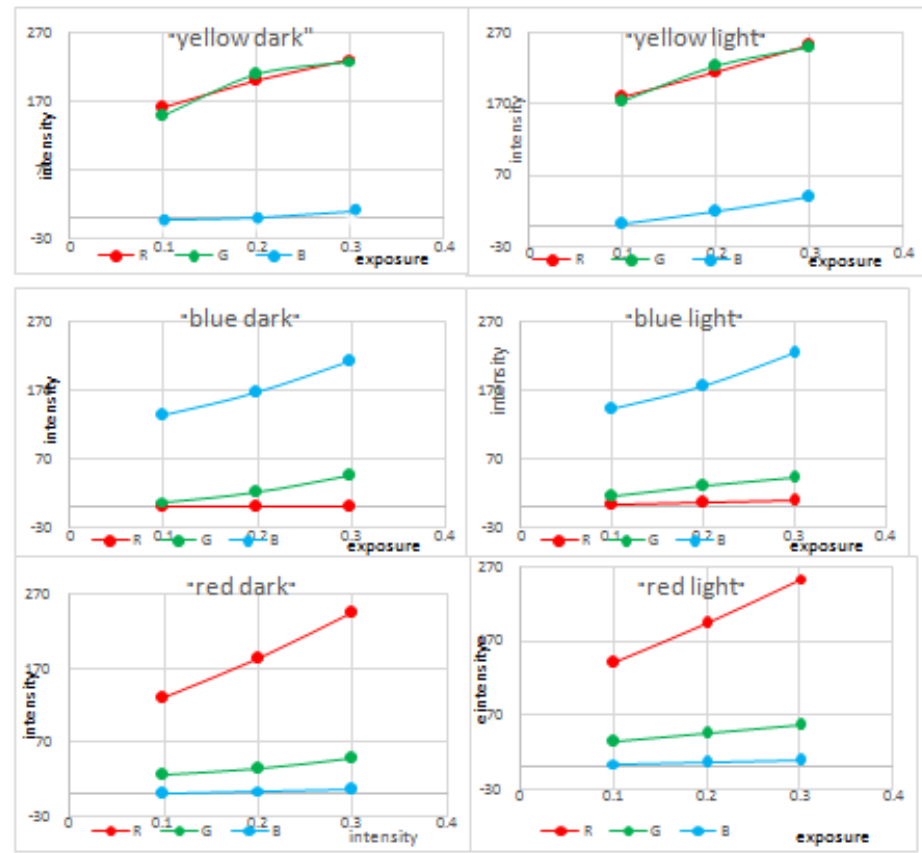

Figure5. Graphs of dependences of the intensities $R, G, B$ on exposure time obtained during processing of digital images of reference samples

The color differences for each pair of reference samples are shown in table 2

Table2. Experimental color differences

\begin{tabular}{|c|c|c|c|c|c|c|c|c|c|}
\hline \multirow{3}{*}{ Exposuretime } & & & & & & & & & \\
\hline & \multicolumn{3}{|c|}{ yellow } & \multicolumn{3}{|c|}{ blue } & \multicolumn{3}{|l|}{ red } \\
\hline & $\Delta \mathrm{R}$ & $\Delta \mathrm{G}$ & $\Delta \mathrm{B}$ & $\Delta \mathrm{R}$ & $\Delta \mathrm{G}$ & $\Delta \mathrm{B}$ & $\Delta \mathrm{R}$ & $\Delta \mathrm{G}$ & $\Delta \mathrm{B}$ \\
\hline 0,1 & 17 & 22 & 2 & 3 & 8 & 9 & 21 & 9 & 3 \\
\hline 0,2 & 14 & 12 & 16 & 6 & 9 & 10 & 11 & 11 & 2 \\
\hline 0,3 & 19 & 20 & 28 & 10 & 11 & 11 & 11 & 8 & 2 \\
\hline
\end{tabular}


Colorimetric research of the self-luminous objects in Software and Hardware environments by the Method of multidimensional scales implementation

Verification of the results for metrological compatibility according to the Cochran and Grubbs criteria in accordance with [] revealed no outlier results. The standard deviation of repeatability was $\mathrm{s}_{\mathrm{R}} \approx$ 0,045 chromaticity units. The standard deviation of intra-laboratory reproducibility, calculated according to [], was $\mathrm{s}_{\mathrm{w}} \approx 0,046$ chromaticity units. The results have shown almost linear dependences in the active part of the dynamic range presented in the figure5, that can be used in the future to build the reference scales.

\section{CONCLuSion}

Digital image processing technologies allow achieving the expansion of the dynamic range of colorimetric measurements and overcoming the limited color coverage of the information and measurement channel elements by digital registration with incrementally increasing exposure time of the object control point and certified reference samples. Calibration dependences of intensity on reference samples brightness and exposure time for three color channels allow determining the color coordinates of the control point by pairing calibration dependences corresponding to different digital images

The process of constructing a colorimetric reference scale is to determine the color coordinates of the digital image in the RGB color space, classify and identify their location in the XYZ color space, build calibration dependences of the color channels intensity on the brightness and exposure time and to determine the chromaticity coordinates. Laboratory technology increases the resolution of color measurement by fixing the nominal quantization steps in the color channels of images and reduces the methodological component of uncertainty.

Any registered real object can have an almost infinite number of virtual implementations. "Almost" in this case means that the number of repeated experiments is limited by the laboratory resources. The correct use of vision systems as measuring devices in colorimetry of software and hardware environments involves ensuring the conditions of measurements unity. It is achieved by constructing reference brightness scales of computer images in color channels and fixing reference points of metrological traceability on them. We offered to use equally bright surfaces of self-luminous objects such as organic LEDs or video terminals as such reference points.

Modern vision systems provide high spatial and brightness resolution within the active area of the dynamic range because so can be used for colorimetric studies of extended objects providing the ability to perform color measurements. Relative extended uncertainty does not exceed $\pm 1 \sim 8 \%$. The developed methodology of colorimetry in hardware and software environments also provides the ability to monitor the characteristics of objects in real time and document the results of studies that can be of great importance in the operational control and confirmation of objects compliance.

The proposed method allows to increase the efficiency and quality of measurements due to the fact that it provides the opportunity to perform measurements in both transmitted and reflected light, simultaneously at all points of the object, which may significantly (by several orders of magnitude) differ in their brightness characteristics. The method can be used in studies of extended luminous objects which characteristics change over time.

\section{REFERENCES}

[1] International Lighting VocabularyCIE) CIE S 017/E: 2011.

[2] Wyszecki, G. Current developments in colorimetry, AIC Color 73, 1973., p. 21-51.

[3] Sorokin, D.A. Abstract of dissertation «Gradation of color difference and identification of porcelain by multidimensional scaling methods» on competition of a scientific degree PhD. Russian Academy of Economics G. V. Plekhanov. 2009. Moscow. -23 p.

[4] Sendel, E. Colorimetric methods for the determination of metals traces. Mir, Moscow 1964 - 90p.

[5] Parmasyan, E.S. Abstract of dissertation on competition of a scientific degree $\mathrm{PhD}$ "Colorimetry and polarimetry of some comentary nebulae". Byuracan Astrophysical Observatory of the Academy of Sciences (Armeniya). Yerevan State University. 1963. Yerevan. -10 p.

[6] Fairchild, M.D. Color Appearance Models. 2th Edition// Rochester Institute, Mansell Color Science Laboratory. 2006. -439 p.

[7] The Validation Model of Information Measuring Channel in Technical Vision Systems/ Yauheniya Saukova / International Journal of Advanced Engineering and Technology / ISSN: 2456-7655 / Impact Factor: RJIF 5.54 / www.newengineeringjournal.com / Volume 1; Issue 4; September 2018; Page No. 28 -33. 
Colorimetric research of the self-luminous objects in Software and Hardware environments by the Method of multidimensional scales implementation

[8] Saukova, Y. The main provisions of the High-Resolution Colorimetry/ Materils of the 11thInternational Scientific and Technical Conference"Instrument Engineering -2018" (14-16 November, 2018, Minsk, Belarusian National Technical University). Available in Russian.Pp. 152-154.

[9] Goden,J. Colorimetric in the rendering performance. Available in Russian. Moscow: Technosphere, 2008. $-328 \mathrm{p}$.

[10] The Metrological Assurance of the Colorimetry in Software and Hardware Environments/ Y. Saukova, I. Matyush/ International Journal of Innovative Research in Electronics and Communications (IJIREC) Volume 3, Issue 5, 2016, PP 6-19 ISSN 2349-4042 (Print) \& ISSN 2349-4050 (Online) DOI: http://dx.doi.org/10.20431/2349-4050.0305002 www.arcjournals.org.

[11] Computer program "Photon". Authors -Saukova, Y.,Matiyush, I.Copyright holder of the computer program isBelarusian National Technical University. 2016. Available in Russian.Registration certificate № 009. Date of entry in the registry of service computer programs 01.03.2017.

[12] Method for measuring the brightness of non-self-luminous objects using matrix photodetectors/Saukova, Y,Zuikov, I. / Materials of the 9thInternational Scientific and Practical Conference "Modern research and development -2013” (17-25 January 2013, Sofia, Bulgaria).2013. Available in Russian. Vol.27. Mathematics. Physics. Modern information technologies. Pp. 38-42.

[13] Colorimetric measurements based on digital registration with high spatial resolution /Saukova, Y, Zuikov, I. / Materials of the 6th International Scientific and Technical Conference"Instrument engineering-2013", Belarusian National Technical University. Available in Russian.Pp. 176-178.

[14] Meshkov,V.V., Matweev,A.B.Basics of lighting. // Textbook for universities: In two parts.Part 2. Physiological optics and colorimetry. - 2thEdition. - Moscow: Energoatomizdat.Available in Russian.1989. - 432 p.

[15] GOST 13088-67. Colorimetry. Terms, letter designations. - Moscow.State Committee for product quality management and standards. Available in Russian1990-13 p.

[16] ISO 5725-2:1994Accuracy (trueness and precision) of measurement methods and results - Part 2: Basic method for the determination of repeatability and reproducibility of a standard measurement method.-43 p.

[17] ISO 7724-2:1984 Paints and varnishes - Colorimetry -- Part 2: Colour measurement. 16 p.

[18] ISO 5725-4-2002 Accuracy (trueness and precision) of measurement methods and results - Part 4: Basic methods for the determination of the trueness of a standard measurement method. $-22 \mathrm{p}$.

Citation: SaukovaYauheniya, "Colorimetric Research of the Self-Luminous Objects in Software and Hardware Environments by the Method of Multidimensional Scales Implementation." International Journal of Innovative Research in Electronics and Communications (IJIREC), 6(4), pp.25-33. http://dx .doi.org /10.20431/2349-4050.0604003

Copyright: (C) 2019 Authors this is an open-access article distributed under the terms of the Creative Commons Attribution License, which permits unrestricted use, distribution, and reproduction in any medium, provided the original author and source are credited. 